\title{
Conflicto ambiental en Ventanas: Análisis desde una perspectiva de la Doctrina Social de la Iglesia
}

\author{
Kay Bergamini Ladrón de Guevara ${ }^{1}$ \\ Ricardo Irarrázabal Sánchez ${ }^{2}$ \\ Piroska Ángel Hernández ${ }^{3}$
}

Recibido: 5 de enero de 2018 - Aprobado: 30 de marzo de 2018

\begin{abstract}
Resumen
Los principios entregados por la Doctrina Social de la Iglesia (DSI) en materia ambiental pueden constituir una fuente de inspiración que ayude a buscar mecanismos de comprensión y de resolución en relación con los problemas sociales y medioambientales en territorios determinados, como por ejemplo el área industrial de Ventanas en Puchuncaví, Chile. El presente proyecto de investigación pretende aportar desde la identificación y sistematización de las conceptualizaciones medioambientales propuestas por la DSI, para evaluar el accionar del Estado y de las empresas que componen el área industrial de Ventanas. Al mismo tiempo, pretende comprender factores y procesos, por medio de entrevistas y encuestas a actores claves del conflicto y así generar propuestas que permitan avanzar en la solución del problema.
\end{abstract}

Palabras clave: Doctrina Social de la Iglesia, conflictos socioambientales, Ventanas, Puchuncaví

\section{Environmental Conflict in Ventanas: Analysis from the Catholic Social Teaching perspective}

\begin{abstract}
The given principles by the Catholic Social Teaching (DSI) in the environmental matter can constitute a source of inspiration helping to find understanding and resolution mechanisms in relation with the social and environmental
\end{abstract}

\footnotetext{
Chileno. Doctor en Gestión Ambiental, Paisaje y Geografía, Universidad de Barcelona, España. Académico, Instituto de Estudios Urbanos y Territoriales, Pontificia Universidad Católica de Chile. E-mail: kbergani@uc.cl

2 Chileno. LLM en Mineral Law and Policy, Universidad de Dundee, Escocia. Académico, Facultad de Derecho, Pontificia Universidad Católica de Chile. E-mail: rirarrazabal@uc.cl 3 Chilena. Magíster en Asentamientos Humanos y Medio Ambiente, Instituto de Estudios Urbanos y Territoriales, Pontificia Universidad Católica de Chile. E-mail: paangel@uc.cl
} 
problems in determined territories, for example, the Industrial Area of Ventanas in Puchuncaví, Chile. The present research project aims to contribute from the identification and systematization of environmental conceptualizations proposed by the DSI to evaluate actions by the State and the companies composing the industrial area of Ventanas. At the same time, through interviews and surveys, this project intends to understand factors, process, and principal actors in the conflict in order to generate proposals to advance in the solution of environmental problems.

Keywords: Catholic Social Teaching, socio-environmental conflict, Ventanas, Puchuncaví

\title{
Conflito Ambiental em Ventanas: Análise desde uma perspectiva da Doutrina Social da Igreja
}

\begin{abstract}
Resumo
Os princípios entregados pela Doutrina Social da Igreja (DSI) em matéria ambiental podem constituir uma fonte de inspiração que ajude a buscar mecanismos de compreensão e de resolução no que refere aos problemas sociais e do meio ambiente em territórios determinados como, por exemplo, a área industrial de Ventanas em Puchuncaví, Chile. 0 atual projeto de investigação visa contribuir desde a identificação e sistematização das conceptualizações sobre o meio ambiente propostas pela DSI, para avaliar as ações do Estado e das empresas que compõem a área industrial de Ventanas. Ao mesmo tempo visa compreender os fatores e processos por meio de entrevistas e pesquisas a atores chaves do conflito, para assim gerar propostas que permitam avançar na solução do problema.
\end{abstract}

Palavras-chave: Doutrina Social da Igreja, conflitos socioambientais, Ventanas, Puchuncaví

\section{Introducción}

La cuestión ecológica o ambiental se ha constituido en un tema relevante para la Doctrina Social de la Iglesia (DSI), especialmente con la última encíclica del Papa Francisco, Laudato Si', la cual, mediante un mensaje simple, intenta sensibilizar a la población mundial respecto del desafío de proteger "nuestra casa común", invitando a una serie de diálogos para superar la crítica situación actual de deterioro ambiental.

La temática ya estaba presente en la DSI, tal como se encuentra reflejado en el Compendio de Doctrina Social de la Iglesia, el que indica que "La responsabilidad de salvaguardar el medio ambiente, patrimonio común del género humano, se extiende no solo a las exigencias del presente, sino también a las del futuro" (Consejo Pontificio Justicia y Paz, 2004: 
punto 473). El mensaje también incentiva a tomar acciones, puesto que "la solución del problema ecológico exige que la actividad económica respete mejor el medio ambiente, conciliando las exigencias del desarrollo económico con las de la protección ambiental" (Consejo Pontificio Justicia y Paz, 2004: punto 470).

El Papa Benedicto XVI (2009), puso especial énfasis en la "Ecología Humana", indicando en el punto 51 de su encíclica Caritas in Veritate que:

"Cualquier menoscabo de la solidaridad y del civismo produce daños ambientales, así como la degradación ambiental, a su vez, provoca insatisfacción en las relaciones sociales. La naturaleza, especialmente en nuestra época, está tan integrada en la dinámica social y cultural que prácticamente ya no constituye una variable independiente".

Por su parte, el Papa Francisco, en una Audiencia General del 5 de junio del 2013, señalaba que:

"Los Papas han hablado de ecología humana, estrechamente ligada a la ecología medioambiental. Nosotros estamos viviendo un momento de crisis; lo vemos en el medio ambiente, pero sobre todo lo vemos en el hombre [...]. Y el peligro es grave porque la causa del problema no es superficial, sino profunda: no es solo una cuestión de economía, sino de ética y de antropología".

Lo anterior resulta interesante de mostrar, a la luz de la reciente publicación de Laudato Si', encíclica papal centrada ampliamente en el conflicto ambiental y sus implicancias sociales (Papa Francisco, 2015).

Uno de los territorios de Chile en que la temática ha tomado significancia, por los problemas de contaminación que han desencadenado un proceso de conflictividad ambiental, corresponde al Parque Industrial de Ventanas (PIV), ubicado en las comunas de Puchuncaví y Quinteros, en la Región de Valparaíso. El PIV fue inaugurado en 1961, con el fin de generar un polo de desarrollo económico en dicho lugar, en un momento en que la consolidación industrial era una de las grandes preocupaciones del Estado chileno, ya que se estimaba que los beneficios que traería eran ampliamente superiores para el progreso que las consecuencias ambientales que pudiesen acarrear para la zona (Correa, 2012). No obstante, a inicios de los años setenta, esta percepción optimista comenzó a ser cuestionada por quienes vivían en el sector, debido a los efectos de los gases emanados desde la fundición, que fueron afectando las actividades agrícolas, ganaderas y la pesca local (Contreras, 2014; Observatorio Latinoamericano de Conflictos Ambientales OLCA, 2012).

Hoy en día, a más de cincuenta años de la fundación del PIV, la situación de deterioro ambiental se ha constituido en un caso paradigmático 
de inequidad ambiental y de afectación a los derechos humanos (Hervé et al., 2012). En efecto, los altos niveles de contaminación evidenciados en diversos estudios científicos (Salmanighabeshi et al., 2015; Gonzalez et al., 2015; De Gregori et al., 2003; Ginocchio, 2000) se traducen, por un lado, en un constante desmedro económico, debido al impacto provocado en los diversos medios de producción de la zona (agricultura, ganadería y pesca) y, por otro, en el daño a la salud de los habitantes que la circundan, quienes han tenido que soportar las cargas ambientales provenientes de la falta de planificación ambiental en el área (Hervé et al, 2012; Vallejo, 2012; Siegel y Siegel, 1995). En este contexto, Ventanas, lugar donde se han instalado más industrias que contribuyen a la contaminación general de la zona, se presenta como un territorio de conflicto, debido al desgaste de las relaciones ocurridas entre las actividades económicas de carácter industrial con las comunidades locales y sus actividades económicas tradicionales, pero también con aquellos vecinos más recientes que han llegado a las zonas aledañas producto del turismo.

Los principios entregados por la Doctrina Social de la Iglesia (DSI) en materia ambiental pueden constituir una fuente de inspiración que ayude a buscar mecanismos de comprensión y de resolución con vínculo a los problemas sociales y medioambientales en territorios determinados, como por ejemplo el área industrial de Ventanas. Es por esto que el presente proyecto de investigación busca aportar desde la identificación y sistematización de las conceptualizaciones medioambientales propuestas por la DSI, para evaluar el accionar del Estado y de las empresas que componen el área industrial, comprender factores y procesos, y así generar propuestas que permitan avanzar en la solución del problema.

Es relevante mencionar, además, que existen diversos análisis de los efectos que la contaminación atmosférica y los pasivos ambientales pueden generar en la salud de las personas, así como en el suelo y la vegetación, en los que se ha podido demostrar la relación entre las actividades económicas, principalmente la fundición de cobre y la contaminación en el área (Parra et al., 2014; Fuenzalida y Quiroz, 2012; Neaman et al., 2009; Oyarzún, 2010). En contraposición a lo anterior, los estudios que abordan la temática ambiental desde una perspectiva social resultan más bien escasos, centrándose en aspectos de conflictividad y justicia ambiental (Sabatini et al., 1997; Cordero, 2011), en los que se da cuenta del desbalance entre la población residente en el área en términos socioeconómicos y la presencia de industrias contaminantes, así como de la ineficacia de los instrumentos de gestión administrativos para resolver los problemas ambientales y sociales existentes.

Conforme lo anterior, la encíclica Caritas in Veritate dedica una sección completa a tratar el tema del desarrollo de los pueblos y el medio ambiente. 
Es importante la visión que entrega respecto de la relación entre el hombre y el ambiente natural, en la que indica que la naturaleza es parte de la obra creadora por Dios, entregada para satisfacer sus necesidades, pero respetando su equilibrio, inherente a su creación. Así, entonces, resulta un aporte al estado del conocimiento en la materia realizar un estudio desde la perspectiva social y ambiental que involucre una mirada de la DSI, pero desde una visión aplicada. En ese contexto los objetivos generales de este estudio son:

1. Identificar y analizar los principios que emanan de la DSI en relación con la problemática ambiental y de sostenibilidad.

2. Caracterizar el área industrial del sector de Ventanas, comuna de Puchuncaví y Quintero, respecto de las empresas existentes, los impactos ambientales generados y las relaciones con las comunidades vecinas.

3. Evaluar y clasificar la gestión ambiental y social del Estado y de las empresas del sector de Ventanas, a la luz de los principios de la DSI en materia ambiental.

\section{Documentos y métodos}

Poder comprender un conflicto ambiental desde la perspectiva de la DSI implica inicialmente la necesidad de contar con una herramienta que se construya a partir de la identificación y clasificación de aquellos atributos o categorías, y de su sistematización posterior que se extraiga. En una primera fase se realizaron dos actividades: una relativa a la caracterización del área industrial de Ventanas y otra correspondiente a la sistematización de la DSI para la generación de principios que permitieron la construcción del instrumento de medición. En una segunda fase se realizaron entrevistas semiestructuradas en terreno a distintos actores que se consideraron relevantes para el estudio. Finalmente, la última fase consideró el análisis de las entrevistas y la generación de propuestas vinculadas. A continuación, se describe en detalle cada una de las actividades indicadas.

Respecto de la caracterización del Área Industrial de Ventanas (Actividad 1), se realizó a partir de una revisión de fuentes secundarias, desde la cual se extrajo, para cada uno de los componentes ambientales, estudios que permitieran confirmar que el área se encuentra contaminada respecto de efectos derivados de la actividad que ahí se desarrolla. Adicionalmente se identificó a cada una de las empresas ahí existentes y las actividades que desarrollan. Esto permitió comprender el contexto del área industrial y el conflicto socioambiental, la evolución de éste y aquellos aspectos inherentes al área que la individualizan respecto de otras. 
En relación con la sistematización de la DSI (Actividad 2), se realizó a partir de la revisión de diversos documentos del magisterio de la iglesia, que incluyó un total de dieciocho, incluyendo cinco encíclicas papales, un compendio de la DSI, cuatro mensajes en jornadas mundiales y cuatro intervenciones papales en otros contextos.

Cinco encíclicas papales:

- Pablo VI (1967). Carta encíclica "Populorum Progressio", dedicada a la necesidad de promover el desarrollo de los pueblos.

- Juan Pablo II (1991). Carta encíclica "Centesimus Annus", con ocasión del Centenario de la encíclica "Rerum Novarum". En ella Juan Pablo II expone su preocupación por el crecimiento de los problemas antiguos, así como los que emergen en el umbral del tercer milenio.

- Juan Pablo II (1995). Carta encíclica "Evangelium Vitae", se centra sobre el valor y el carácter inviolable de la vida humana.

- Benedicto XVI (2009). Carta encíclica "Caritas in Varitate", sobre el desarrollo humano integral en la caridad y en la verdad.

- Francisco (2015). Carta encíclica "Laudato Si'", centrada en el cuidado de la casa común, el planeta Tierra.

Un compendio de DSI:

- Juan Pablo II (2004). Compendio de la Doctrina Social de la Iglesia, una compilación de todas las enseñanzas sociales de la Iglesia católica.

Cuatro exhortaciones apostólicas:

- Juan Pablo II (1988). Exhortación Apostólica Postsinodal "Christifideles Laici", sobre vocación y misión de los laicos en la iglesia y en el mundo.

- Juan Pablo II (1999a). Exhortación Apostólica Postsinodal "Ecclesia in Asia", para la expansión de la fe católica en Asia.

- Juan Pablo II (1999b). Exhortación Apostólica Postsinodal "Ecclesia in América", que da camino para la conversión, la comunión y la solidaridad en América.

- Juan Pablo II (2003). Exhortación Apostólica Postsinodal Pastores Gregis.

Cuatro mensajes en jornadas mundiales:

- Mensaje de Su Santidad Juan Pablo II para la celebración de la XXIII Jornada Mundial de la Paz (1990). 
- Mensaje del Santo Padre Juan Pablo II para la Jornada Mundial de la Alimentación (2004).

- Mensaje de Su Santidad Benedicto XVI para la celebración de la XLII Jornada Mundial de la Paz (2009).

- Mensaje de Su Santidad Benedicto XVI para la celebración de la XLIII Jornada Mundial de la Paz (2010).

Cuatro intervenciones papales en otros contextos:

- Papa Francisco, Audiencia General (5-VI-2013).

- Conferencia Episcopal Paraguaya, Carta Pastoral "El campesino paraguayo y la tierra" (1983).

- Declaración Conjunta del Santo Padre Juan Pablo II y su santidad Bartolomé I. Firma de la Declaración de Venecia (2002).

- Discurso del Santo Padre Juan Pablo Il a los promotores y participantes en un Congreso Internacional sobre "Ambiente y Salud" (1997).

La revisión se realizó clasificando los párrafos de dichos documentos en función de elementos comunes del discurso que permitieran la identificación de "principios". Una vez realizada la sistematización, a partir de los principios observados, se procedió a construir el instrumento de pauta de entrevista semiestructurada.

Finalizada la caracterización y construida la pauta de entrevista, se procedió a su aplicación en terreno. Inicialmente, es importante indicar que la selección de la muestra para esta investigación se consideró de tipo cualitativa no aleatoria, delimitada a partir de los actores involucrados directamente con el conflicto ambiental del sector industrial de Ventanas. Esta muestra estuvo compuesta por: empresarios del sector industrial en el área de estudio; autoridades ambientales de la región de Valparaíso, autoridades locales de Puchuncaví y Quintero, y actores claves de la comunidad, tales como dirigentes de organizaciones territoriales o funcionales que tuvieran vinculación con el área. No se seleccionan otros actores que se podrían llamar "externos", es decir, que no tuvieran vinculación con el sector industrial de Ventanas, debido a que ellos podrían aportar con una percepción indirecta obtenida principalmente a partir de medios de comunicación, lo cual, en esta investigación, no resulta del todo relevante. El criterio utilizado para determinar el número de actores a entrevistar es el de saturación, es decir, una vez que los testimonios ya no entreguen nuevos elementos y se presenten en ellos discursos reiterativos, la recolección de información cesa. En total se realizaron catorce entrevistas a actores relevantes, que correspondieron a tres empresas, tres autoridades y ocho a representantes de la comunidad. La aplicación de las entrevistas 
en terreno se realizó de manera presencial, mediante un contexto de conversación informal y registrándolas a través de grabaciones y notas de campo, las cuales posteriormente se transcribieron.

Finalmente, la última actividad de análisis (Actividad 4) se realizó a partir de la sistematización de las transcripciones de las entrevistas, clasificándolas en cada uno de los principios de DSI identificados previamente. A su vez, se mantuvo la categorización del origen de cada uno de los actores relevantes, con lo que se configuró una matriz de análisis que permitió comparar visiones respecto de temas comunes.

\section{Caracterización del área industrial sector de Ventanas, comuna de Puchuncaví}

La comuna de Puchuncaví fue creada en 1944 después de una prolongada movilización para separarse de la comuna de Quinteros, y así adquirir mayor autonomía. Durante los primeros años, la comuna se sustentaba principalmente en la agricultura, siendo los principales productos sus cultivos de lenteja, garbanzo, arveja y maíz. La actividad para el año 1952 mantenía una tasa de ocupación comunal del 75\%. La pesca, poco a poco, comenzó a tener mayor protagonismo, y para las décadas de 1950 y 1960 tanto la agricultura como la pesca eran parte fudamental de la economía local (Calisto, 2014).

Por otra parte, el desarrollo industrial de la localidad de Ventanas, ubicada en la comuna de Puchuncaví, se sitúa a fines de la década de 1950 con el funcionamiento de la empresa Chilectra, que más tarde pasa a llamarse AES GENER. Luego, en 1964, se inaugura la fundición de cobre de la Empresa Nacional de Minería y entra en operación la central termoeléctrica Ventanas I (Comisión de Recursos Naturales, Bienes Nacionales y Medio Ambiente, 2011). La instalación del complejo industrial representó un importante motivo de movilización de la sociedad política de Valparaíso, dado que en ese entonces las comunidades competían entre sí por conseguir para sus comunas la nueva fundición de cobre y el ansiado "polo de desarrollo regional". La disputa por localizar el complejo industrial no solo se limitó a consideraciones económicas y la creación de empleos, sino que incluyó debates sobre defensa nacional, facilidades de transporte e impactos de la contaminación. Este útimo factor fue el decisivo para su instalación en la localidad de Ventanas, debido a que los informes técnicos de la época consideraban que la zona presentaba mejores condiciones para la dispersión de gases, además de tener poco o ningún efecto sobre la economía y la salud en la localidad. En este contexto, finalmente se establece el complejo industrial de Ventanas, privilegiando los intereses de la región y el país por 
sobre la localidad rural, que terminaría por deteriorar gravemente la calidad de los suelos, sus economías tradicionales y la salud de la comunidad (Sabatini et al., 1997). Hoy, el Parque Industrial se compone de una red de empresas que se enfocan principalmente en el ámbito energético nacional (ver figura 1).

\section{Figura 1}

Empresas del Parque Industrial de Ventanas

\begin{tabular}{|l|l|l|}
\hline NOMBRE & RUBRO \\
\hline AES Gener & Generación de energía a carbón \\
\hline Catamutún & $\begin{array}{l}\text { Importación, cribado y distribución de } \\
\text { carbón }\end{array}$ \\
\hline $\begin{array}{l}\text { Puerto } \\
\text { Ventanas }\end{array}$ & Recepción de materias primas \\
\hline Melón & $\begin{array}{l}\text { Almacenamiento de materiales para } \\
\text { elaboración de cemento }\end{array}$ \\
\hline Codelco & $\begin{array}{l}\text { Fundición y refinería de cobre, oro y } \\
\text { plata }\end{array}$ \\
\hline Oxiquim & Terminal de descarga graneles líquidos \\
\hline Gasmar & $\begin{array}{l}\text { Recepción, elaboración y } \\
\text { comercialización gas licuado del } \\
\text { petróleo }\end{array}$ \\
\hline ENAP & $\begin{array}{l}\text { Almacenamiento y transporte de } \\
\text { Petróleo }\end{array}$ \\
\hline GNL & $\begin{array}{l}\text { Almacenamiento de gas y } \\
\text { regasificación }\end{array}$ \\
\hline Shell - Enex & Planta de lubricantes \\
\hline COPEC & Planta de lubricantes \\
\hline ENDESA & Generación de energía \\
\hline
\end{tabular}

Fuente: Elaboración propia

Esta zona se encuentra contaminada por la acumulación de emisiones de las empresas que operan en el territorio, las cuales han generado impactos en las diferentes componentes ambientales, principalmente en las localidades aledañas a la zona industrial: Las Ventanas, La Greda y Maitenes (Correa, 2012; Contreras, 2014; Observatorio Latinoamericano de Conflictos Ambientales OLCA, 2012). De acuerdo con distintos autores, el área se encuentra contaminada en:

Aire: Se presenta primordialmente a través de contaminación por material particulado y ruido. Los principales elementos químicos identificados son: Plomo, Arsénico, Selenio, Cobre, Molibdeno, Vanadio y Cadmio (Correa, 2012; Sánchez, 2013), siendo la Estación de monitorio La Greda la que lidera las concentraciones más importantes de Cobre, Cadmio y Molibdeno, y la segunda 
Estación con las concentraciones más elevadas de Arsénico, de acuerdo con el Informe de Calidad del Aire 2009 (Sánchez, 2013; Observadores de Derechos Humanos, Casa Memoria José Domingo Cañas, 2013). La problemática atmosférica se reconoce como la más urgente, especialmente por eventos tales como la intoxicación masiva de los niños de La Greda o el alto índice de enfermedades broncopulmonares en las comunidades aledañas (Calisto, 2014).

Agua de mar: Se ha determinado la presencia de metales pesados e hidrocarburos (Sánchez, 2013). Por otra parte, las tuberías que recirculan el agua de enfriamiento de las turbinas de las centrales térmicas, modifican la temperatura del agua lo cual puede causar: daños a la flora y fauna, aumento del crecimiento de bacterias y otros organismos, aceleración de reacciones químicas, reducción de los niveles de oxígeno disuelto y daño a los organismos vivos (Observadores de Derechos Humanos, Casa Memoria José Domingo (añas, 2013). A la vez, se identifica la presencia de grasas y aceites que inciden en la reducción de la transmisión de la luz, disminución de oxígeno disuelto, daños a la flora y fauna acuática. También presenta sólidos suspendidos, los cuales causan la pérdida de la fauna acuática, merma de la penetración de la luz en el agua y enturbiamiento del agua. Los cambios de PH en el agua de mar causa perturbaciones celulares en los organismos vivos y la destrucción de la biodiversidad acuática (Observadores de Derechos Humanos, Casa Memoria José Domingo Cañas, 2013).

Suelo: Se ha encontrado Cadmio, Cobre, Plomo, Cromo, Arsénico, Níquel y Selenio (Sánchez, 2013). De acuerdo con las distintas normas (Suiza, Canadá y Alemania), con las cuales se diferencian los suelos comerciales, industriales y residenciales en función de la presencia de ciertos contaminantes, los suelos de la localidad de La Greda tendrían características de industrial, conforme la presencia de ciertos parámetros que excederían los valores referenciales. A la vez se analizaron 14 establecimientos educacionales y en todos ellos se estableció la presencia de contaminación de metales pesados en distintos grados (Observadores de Derechos Humanos, Casa Memoria José Domingo Cañas, 2013).

Biodiversidad: El año 2012 la ONG Oceana realizó un muestreo en mariscos y crustáceos (almejas, lapas, locos y jaibas) cerca de la localidad de Ventanas, el estudio posterior revela contaminación con Cobre, Arsénico y Cadmio en el $100 \%$ de las especies analizadas (ONG Oceana, 2012).

Medio Humano: Se manifiesta principalmente a través de las enfermedades derivadas de la contaminación ambiental, tales como cáncer, problemas respiratorios y dermatológicos, también enfermedades mentales o cardiovasculares, entre otras (Sánchez, 2013). La contaminación industrial también ha afectado las actividades económicas tradicionales de este sector, como la pesca artesanal. Los pescadores de Ventanas han debido cambiar su oficio al de recolectores de alga frente a la escasez de peces y mariscos (Sánchez, 2013). 


\section{Principios de la DSI y medio ambiente}

De la revisión de los distintos textos del magisterio de la iglesia se logró determinar seis principios de la DSI de primer orden y ocho principios de la DSI de segundo, permitiendo estos últimos complementar los primeros:

1. Principio de la Responsabilidad.

2. Principio de la Tutela del Medio Ambiente.

3. Principio de la Ecología Integral y de la Ecología Humana.
i. Principio del destino universal de los bienes.
ii. Principio de la tutela de los derechos indígenas.
iii. Instrumento de Paz social.

4. Principio Conciliador Desarrollo y Cuidado medioambiental.
i. Principio de la Gestión Ambiental.
ii. Principio Preventivo y Correctivo.

5. Principio de la Solidaridad.
i. Principio de la Solidaridad Intergeneracional.
ii. Principio de la Solidaridad Universal.

6. Principio Precautorio y de Ciencias.

A continuación, se describe cada uno de los principios de primer orden identificados:

1. Principio de la Responsabilidad: Es el principio rector de la Doctrina Social de la Iglesia (DSI) en materia del medio ambiente y del cual derivan el resto de los principios, el cual tiene su origen en la conceptualización del ambiente natural como un "don de Dios para todos, y su uso representa para nosotros una responsabilidad para con los pobres, las generaciones futuras y toda la humanidad" (Benedicto XVI, 2009: punto 48)

2. Principio de la Tutela del Medio Ambiente: Del principio de la "responsabilidad" del ser humano con la creación, surge entonces un deber de tutela ambiental. De acuerdo con Laudato Si', "implica que el ser humano, dotado de inteligencia, respete las leyes de la naturaleza y los delicados equilibrios entre los seres de este mundo (Papa Francisco, 2015: punto 8), cuestión que remarca el Compendio, al indicar que "la tutela del medio ambiente constituye un desafío para la entera humanidad: se trata del deber, común y universal, de respetar un bien colectivo, destinado a todos" (Consejo Pontificio Justicia y Paz, 2004: punto 466).

3. Principio de la Ecología Integral y de la Ecología Humana: La "Ecología Humana" y la "Ecología Integral" están enraizadas en el principio 1 de la 
Declaración de Río en la Cumbre de la Tierra (1992), el cual establece la primacía de la persona como centro de las preocupaciones del DesarroIlo Sustentable. Tanto el Papa San Juan Pablo II como el Papa Benedicto XVI hicieron claras alusiones a la "Ecología Humana". En efecto, en un discurso de San Juan Pablo II a la Oficina Europea de Medio Ambiente en 1996, el Pontífice declara que:

"La Iglesia Católica está atenta a la conservación y a la protección del medio ambiente, así como a los problemas que conciernen al desarrollo, según su propia perspectiva antropológica, compartida por los hombres de buena voluntad y por las nobles tradiciones religiosas. Tanto el medio ambiente como el desarrollo se relacionan con la persona humana, centro de la creación. Además, las decisiones económicas y políticas en materia de medio ambiente han de tomarse para servir a las personas y a los pueblos".

Por su parte, el Papa Benedicto XVI en Caritas in Veritate, señala que:

"Es lícito que el hombre gobierne responsablemente la naturaleza para custodiarla, hacerla productiva y cultivarla también con métodos nuevos y tecnologías avanzadas, de modo que pueda acoger y alimentar dignamente a la población que la habita. En nuestra tierra hay lugar para todos: en ella toda la familia humana debe encontrar los recursos necesarios para vivir dignamente, con la ayuda de la naturaleza misma, don de Dios a sus hijos, con el tesón del propio trabajo y de la propia inventiva. Pero debemos considerar un deber muy grave el dejar la tierra a las nuevas generaciones en un estado en el que puedan habitarla dignamente y seguir cultivándola. Eso comporta el compromiso de decidir juntos después de haber ponderado responsablemente la vía a seguir, con el objetivo de fortalecer esa alianza entre ser humano y medio ambiente que ha de ser reflejo del amor creador de Dios, del cual procedemos y hacia el cual caminamos". (Benedicto XVI, 2009: punto 50)

Este concepto de ecología humana y la centralidad social de la misma es tomado por el Papa Francisco en Laudato Si al plantear la "Ecología Integral" en el punto 137, cuando remarca que:

"Dado que todo está íntimamente relacionado, y que los problemas actuales requieren una mirada que tenga en cuenta todos los factores de la crisis mundial, propongo que nos detengamos ahora a pensar en los distintos aspectos de una ecología integral, que incorpore claramente las dimensiones humanas y sociales".

4. Principio Conciliador Desarrollo y Cuidado medioambiental: El principio "conciliador" tiene que ver con la forma como debiera implementarse el principio de la Responsabilidad y el de la Ecología Integral, esto es, cómo 
armonizar el desarrollo con el cuidado ambiental, pero con especial énfasis en los problemas humanos y sociales que derivan de la problemática ambiental. Este principio ya estaba señalado en el Compendio, cuando mencionaba que,

"La solución al problema ecológico exige que la actividad económica respete mejor el medio ambiente, conciliando las exigencias del desarrollo económico con las de la protección ambiental. Cualquier actividad económica que se sirva de los recursos naturales debe preocuparse también de la salvaguardia del medio ambiente y prever sus costos, que se han de considerar como un elemento esencial del coste actual de la actividad económica". (Consejo Pontificio Justicia y Paz, 2004: punto 470)

En Caritas in Veritate el Papa Benedicto XVI se hace cargo de esta temática, al mencionar en forma indirecta el principio ambiental "contaminadorpagador" y lo que significa el proceso de internalización de las externalidades negativas, tanto ambientales como sociales:

"Las autoridades competentes han de hacer los esfuerzos necesarios para que los costes económicos y sociales que se derivan del uso de los recursos ambientales comunes se reconozcan de manera transparente y sean sufragados totalmente por aquellos que se benefician, y no por otros o por las futuras generaciones". (Benedicto XVI, 2009: punto 50)

Por otra parte, en el punto 190 de la encíclica Laudato Si', el Papa Francisco se cuestiona:

"¿Es realista esperar que quien se obsesiona por el máximo beneficio se detenga a pensar en los efectos ambientales que dejará a las próximas generaciones? Dentro del esquema del rédito no hay lugar para pensar en los ritmos de la naturaleza, en sus tiempos de degradación y de regeneración, y en la complejidad de los ecosistemas".

Para entender a cabalidad el principio "conciliador", resulta esencial comprenderlo a la luz del principio preventivo y precautorio, en relación con la gestión ambiental que se proponga. En este sentido, la encíclica Laudato Si es clara al señalar que "Cuando aparecen eventuales riesgos para el ambiente que afecten al bien común presente y futuro, esta situación exige que las decisiones se basen en una comparación entre los riesgos y los beneficios hipotéticos que comporta cada decisión alternativa posible" (Papa Francisco, 2015: punto 184).

5. Principio de la Solidaridad: El principio de la Solidaridad nace como una forma de extender el principio Conciliador en relación con las futuras generaciones y desde una conceptualización universal. En efecto, este principio 
tiene dos vertientes, la primera referida a la Solidaridad Internacional en temas ambientales y sociales. Tal como se destaca en Caritas In Veritate, "Hay también una urgente necesidad moral de una renovada solidaridad, especialmente en las relaciones entre países en vía de desarrollo y países altamente industrializados" (Benedicto XVI, 2009: punto 49). Así, "la protección del entorno, de los recursos y del clima requiere que todos los responsables internacionales actúen conjuntamente y demuestren prontitud para obrar de buena fe, en el respeto, la ley y la solidaridad con las regiones más débiles del planeta" (Benedicto XVI, 2009: punto 50). Este principio se relaciona con el principio de las responsabilidades comunes pero diferenciadas, en el sentido de que:

"Sigue siendo cierto que hay responsabilidades comunes pero diferenciadas, sencillamente porque, como han dicho los Obispos de Bolivia, los países que se han beneficiado por un alto grado de industrialización, a costa de una enorme emisión de gases invernaderos, tienen mayor responsabilidad en aportar a la solución de los problemas que han causado". (Papa Francisco, 2015: punto 170)

Respecto del cambio climático, esta situación se vuelve especialmente compleja ya que:

"Las regiones y los países más pobres tienen menos posibilidades de adoptar nuevos modelos en orden a reducir el impacto ambiental, porque no tienen la capacitación para desarrollar los procesos necesarios y no pueden cubrir los costos. Por eso, hay que mantener con claridad la conciencia de que en el cambio climático hay responsabilidades diversificadas". (Papa Francisco, 2015: punto 52)

Pero no solo la perspectiva internacional es relevante, sino que también el componente intergeneracional para la aplicación efectiva del principio Conciliador. En efecto,

"Los proyectos para un desarrollo humano integral no pueden ignorar a las generaciones sucesivas, sino que han de caracterizarse por la solidaridad y la justicia intergeneracional, teniendo en cuenta múltiples aspectos, como el ecológico, el jurídico, el económico, el político y el cultural". (Benedicto XVI, 2009: punto 48)

6. Principio Precautorio y de Ciencias: En la Encíclica Laudato Si' se señala que dicho principio se basa en la Declaración de Río de 1992, en donde se sostiene que, cuando existe peligro de daño grave o irreversible, la falta de certeza científica no deberá utilizarse como razón para postergar la adopción de medidas eficaces que impidan la degradación y afectación del medioambiente. En este sentido, el principio Precautorio y de Ciencias permite la protección de los más débiles, que disponen de pocos recursos para 
defenderse y aportar con pruebas irrefutables. De esta manera, solo a partir de la demostración objetiva y contundente de que la actividad propuesta no generará daños al ambiente o a quienes lo habitan podrá ser llevada a cabo. En el Compendio de la Doctrina Social de la Iglesia se señala que:

"Las autoridades llamadas a tomar decisiones para hacer frente a los riesgos contra la salud y el medio ambiente, a menudo se encuentran ante situaciones en las que los datos científicos disponibles son contradictorios o cuantitativamente escasos: puede ser oportuno entonces hacer una valoración según el 'principio de precaución', que no comporta la aplicación de una regla, sino una orientación para gestionar situaciones de incertidumbre. Este principio evidencia la necesidad de tomar una decisión provisional, que podrá ser modificada en base a nuevos conocimientos que eventualmente se logren". (Consejo Pontificio Justicia y Paz, 2004: punto 469)

Como conclusión de esta sistematización de los principios ambientales contenidos en la DSI, se puede señalar que el principio de la Responsabilidad juega un rol primordial en la ordenación del resto de los principios. Dicho principio se concreta en el deber de tutela o de protección del medio ambiente, el cual ha de ser llevado a la práctica teniendo especial consideración la problemática socioambiental, y recogiendo los principales conceptos de la "Ecología Integral" y "Ecología Humana". El cuidado se realiza a través del "Principio Conciliador", el cual es matizado por el principio de la Solidaridad, tanto en su vertiente internacional como intergeneracional. A su vez, se considera que las decisiones deben ser tomadas considerando el principio Precautorio y de ciencias, es decir, antes de actuar sobre el medioambiente se debe tener bases irrefutables de que no se producirán daños graves o irreversibles.

\section{Conflicto ambiental en Ventanas desde la perspectiva de la DSI}

Sobre la base de la sistematización de los principios de la DSI en materia de medioambiente y su funcionalidad en entrevistas que buscaban indagar en las percepciones de los actores involucrados en el conflicto ambiental, se realiza el análisis de contenido, en el que se puede obtener lo siguiente:

\section{Principio de la Responsabilidad}

La responsabilidad socioambiental de las empresas tiene matices en su percepción de evaluación por los diferentes grupos entrevistados. Algo en que existe un acuerdo es que los cambios paradigmáticos han repercutido de una u otra forma en la reorientación de las políticas empresariales en su entorno. No obstante, la efectividad de estos cambios es lo que está en discusión. Si bien 
las industrias han incorporado planes, acciones y departamentos dedicados exclusivamente al relacionamiento público y el medioambiente, que ha desencadenado el reconocimiento y valoración de la comunidad, por otra parte, el pasivo ambiental provocado por años de funcionamiento industrial sin normas acordes al cuidado del medioambiente y la salud de los residentes, así como los accidentes industriales, continúan configurando un territorio en crisis, cuya resolución y superación es incapaz de ser abordada con el modelo descrito que se ha utilizado, y que se ha sustentado en las acciones individuales de cada empresa y no todas en su conjunto.

Por otra parte, muchos de estos planes y programas van asociados a proyectos comunitarios que poco se relacionan con soluciones medioambientales, y más bien se transforman en pagos que desarticulan las relaciones comunitarias. A la vez, es cierto que parte de la comunidad reconoce que éstos son claves para abordar la pobreza de la localidad, pero tampoco han sido proyectos que apunten al fortalecimiento del desarrollo local, que entreguen herramientas para su superación, sino más bien son planes paliativos, por lo que las empresas no son claves ni para el mejoramiento de la calidad ambiental ni para el desarrollo económico.

Uno de los grandes problemas que se constituye en torno al PIV es el pasivo ambiental y quién debe responsabilizarse por él. Ni el Estado ni las industrias se han comprometido con su solución, lo que deja de manifiesto la dificultad para plantear avances más concretos en torno a la crisis ambiental. En este sentido, cabe destacar que no se ha tomado responsabilidad frente a la contaminación histórica, ni frente al daño a la microeconomía local. Las percepciones de los actores entrevistados se indican a continuación:

\section{Tabla 1}

Principio de responsabilidad

\begin{tabular}{|l|l|l|}
\hline \multicolumn{1}{|c|}{ Empresas } & \multicolumn{1}{|c|}{ Autoridades } & \multicolumn{1}{c|}{ Comunidad } \\
\hline $\begin{array}{l}\text { No se reconoce un } \\
\text { responsable del conflicto y se } \\
\text { culpa al Pasivo Ambiental. }\end{array}$ & $\begin{array}{l}\text { Responsables son el SEIA, } \\
\text { empresas y comunidad. }\end{array}$ & $\begin{array}{l}\text { Los responsables del conflicto } \\
\text { ambiental son principalmente } \\
\text { las empresas antiguas y el } \\
\text { Estado. }\end{array}$ \\
\hline $\begin{array}{l}\text { No todas las empresas han } \\
\text { profundizado de la misma } \\
\text { manera en torno a las } \\
\text { acciones de responsabilidad } \\
\text { socioambiental. }\end{array}$ & $\begin{array}{l}\text { Empresas nuevas son } \\
\text { más responsables } \\
\text { socioambientalmente que las } \\
\text { más antiguas. }\end{array}$ & $\begin{array}{l}\text { Las empresas nuevas son } \\
\text { más responsables con el } \\
\text { medioambiente, pero menos } \\
\text { involucradas con la comunidad } \\
\text { y la responsabilidad social. }\end{array}$ \\
\hline $\begin{array}{l}\text { No se consideran causantes } \\
\text { del conflicto ambiental. }\end{array}$ & $\begin{array}{l}\text { Nadie se hace cargo del } \\
\text { pasivo ambiental. }\end{array}$ & $\begin{array}{l}\text { Nadie se hace cargo del Pasivo } \\
\text { Ambiental. }\end{array}$ \\
\hline $\begin{array}{l}\text { Las normas son exigentes, } \\
\text { pero tardías. }\end{array}$ & $\begin{array}{l}\text { SElA no ha estado a la altura, } \\
\text { aprobando nuevos proyectos } \\
\text { y con normas poco flexibles y } \\
\text { exigentes. }\end{array}$ & $\begin{array}{l}\text { Presencia débil del Estado en } \\
\text { solucionar el problema. }\end{array}$ \\
\hline
\end{tabular}

Fuente: Elaboración propia 


\section{Principio de la Tutela del Medioambiente}

En relación con el deber de tutela, consecuencia del principio de Responsabilidad, las percepciones evidencian la carencia de implementación de estos principios por parte de las empresas y el Estado, quienes, por medio de sus herramientas y políticas, no han logrado encaminar la solución del conflicto. De hecho, llama la atención que los estudios técnico-científicos han confirmado las negativas consecuencias socioambientales del desarrollo industrial en la zona, lo que sin embargo no se ha traducido en limitaciones respecto del desarrollo de nuevas actividades económicas mediante la aprobación de nuevas superficies de terreno para proyectos industriales. Ello, de alguna manera, exige al Estado un mayor rol y protagonismo para tutelar el medioambiente, poniendo el énfasis en el mejoramiento de la calidad ambiental y social, y aplicando medidas necesarias para ello.

\section{Tabla 2}

Principio de la Tutela del Medioambiente

\begin{tabular}{|c|c|c|}
\hline Empresas & Autoridades & Comunidad \\
\hline $\begin{array}{l}\text { Se hacen estudios técnico- } \\
\text { científicos para asegurar la } \\
\text { actividad económica en el largo } \\
\text { plazo. }\end{array}$ & $\begin{array}{l}\text { Falta de evaluación de } \\
\text { impactos sinérgicos de las } \\
\text { empresas. } \\
\text { Estudios poco claros para la } \\
\text { comunidad. }\end{array}$ & $\begin{array}{l}\text { Estudios que realizan } \\
\text { las empresas son poco } \\
\text { claros y no consideran } \\
\text { el cuidado del } \\
\text { medioambiente. } \\
\text { Desconfianza hacia } \\
\text { estudios de empresas. }\end{array}$ \\
\hline $\begin{array}{l}\text { Hay empresas que solo cumplen la } \\
\text { norma, otras que se adelantan a los } \\
\text { cambios, y las menos que tienen } \\
\text { estándares internacionales. }\end{array}$ & $\begin{array}{l}\text { Solo cumplimiento } \\
\text { normativo básico. }\end{array}$ & $\begin{array}{l}\text { A pesar de problemas } \\
\text { socioambientales en el } \\
\text { territorio, se autorizan } \\
\text { nuevas empresas. }\end{array}$ \\
\hline $\begin{array}{l}\text { Existen tres categorías de empresas } \\
\text { las que se ajustan a la normativa; } \\
\text { las que prevén cambios normativos } \\
\text { y comienzan a cambiar su } \\
\text { tecnología, y las que de manera } \\
\text { inicial de inversión (empresas } \\
\text { nuevas) vienen con tecnologías } \\
\text { mejores, regidas por estándares } \\
\text { internacionales de la empresa. }\end{array}$ & $\begin{array}{l}\text { Mayor incorporación de } \\
\text { tecnología por parte de las } \\
\text { empresas en el tiempo. }\end{array}$ & $\begin{array}{l}\text { Empresas no tienen } \\
\text { disposición a emplear } \\
\text { nuevas tecnologías. }\end{array}$ \\
\hline $\begin{array}{l}\text { Ingreso de profesionales } \\
\text { preocupados por el medioambiente } \\
\text { en las empresas. Departamentos, } \\
\text { programas, etc. }\end{array}$ & $\begin{array}{l}\text { Se destaca el Acuerdo de } \\
\text { Producción Limpia (APL). }\end{array}$ & $\begin{array}{l}\text { Un grupo indica que } \\
\text { se están realizando } \\
\text { proyectos de mejora de la } \\
\text { Bahía de Quintero. }\end{array}$ \\
\hline
\end{tabular}

Fuente: Elaboración propia 


\section{Principio de la Ecología Integral y de la Ecología Humana}

Respecto de este principio, se puede indicar que la percepción de los distintos actores es que la relación entre las empresas y la comunidad, en general, es positiva. Los diversos programas de esparcimiento, educación, becas, financiamiento que ofrecen las industrias a la comunidad, generan un vínculo de valoración. Estas políticas muchas veces son vistas como buena voluntad y responsabilidad de las empresas frente a su entorno; sin embargo, en ocasiones estos proyectos son parte de las medidas de compensación por daños ambientales, es decir, es una obligación su implementación. Por lo tanto, entre mayor impacto de las empresas, son mayores las compensaciones $y$, a la vez, la comunidad va percibiendo mejores políticas de la empresa y una mayor preocupación. Ello se evidencia principalmente tras las dos grandes contingencias ambientales ocurridas en la zona: la nube tóxica y el derrame de petróleo, en las cuales la comunidad percibe que ambos sucesos marcan un antes y un después de la responsabilidad empresarial local; sin embargo, gran parte de sus proyectos corresponden a medidas de compensación y exigencias políticas para responder a la problemática.

Es importante señalar que existen grupos que han visto deteriorarse a lo largo del tiempo sus fuentes laborales, producto de la contaminación, es decir, aquellos que dependen o dependían de los recursos naturales, tales como los pescadores. Ellos mantienen una doble relación con la industria, tanto positiva como negativa, debido a que han experimentado un proceso de menoscabo de su fuente laboral, lo que ha significado la manifestación frente a las empresas y el Estado; sin embargo, la no respuesta ante la problemática los ha situado como dependientes de las empresas del PIV para diversificar, cambiar su fuente laboral o aumentar su producción. 
Tabla 3

Principio de la Ecología Integral y de la Ecología Humana

\begin{tabular}{|l|l|l|}
\hline \multicolumn{1}{|c|}{ Empresas } & \multicolumn{1}{|c|}{ Autoridades } & \multicolumn{1}{c|}{ Comunidad } \\
\hline $\begin{array}{l}\text { Relación con las } \\
\text { comunidades ha sido } \\
\text { positiva. }\end{array}$ & $\begin{array}{l}\text { Aspectos positivos surgen de } \\
\text { política empresarial y relaciones } \\
\text { comunitarias. Se ha centrado en } \\
\text { proyectos. }\end{array}$ & $\begin{array}{l}\text { Se percibe que proyectos que } \\
\text { realizan las empresas son positivos y } \\
\text { por eso existe una buena relación. }\end{array}$ \\
\hline $\begin{array}{l}\text { Incentivo económico } \\
\text { más que el ambiental ha } \\
\text { amortiguado conflictividad } \\
\text { en la zona. }\end{array}$ & $\begin{array}{l}\text { Un aspecto negativo se ve } \\
\text { en que persiste la demanda } \\
\text { por mejora ambiental de las } \\
\text { empresas. }\end{array}$ & $\begin{array}{l}\text { Empresas generan relaciones de } \\
\text { fidelidad con la comunidad, pero no } \\
\text { mejoran problema ambiental. }\end{array}$ \\
\hline $\begin{array}{l}\text { No se identifican } \\
\text { vínculos negativos con la } \\
\text { comunidad. }\end{array}$ & $\begin{array}{l}\text { Existen vínculos positivos y } \\
\text { negativos entre empresas y } \\
\text { comunidad. }\end{array}$ & $\begin{array}{l}\text { Se ve que empresas han dividido a la } \\
\text { comunidad por medio de asignación } \\
\text { de beneficios a familias. }\end{array}$ \\
\hline $\begin{array}{l}\text { No se percibe relación } \\
\text { de conflicto con la } \\
\text { comunidad, destacan los } \\
\text { trabajos en conjunto. }\end{array}$ & $\begin{array}{l}\text { Conflictividad aumenta con } \\
\text { llegada de nuevas empresas. }\end{array}$ & $\begin{array}{l}\text { La comunidad tiene mejor } \\
\text { relación con las empresas que } \\
\text { han sido criticadas por daño } \\
\text { ambiental debido a sus políticas de } \\
\text { relacionamiento. }\end{array}$ \\
\hline
\end{tabular}

Fuente: Elaboración propia

\section{Principio Conciliador Desarrollo y Cuidado medioambiental}

En cuanto a este principio, los entrevistados indican que se buscó, por una parte, ahondar en instancias de diálogo entre los actores involucrados en el conflicto, donde se reconoce que no han dado los resultados esperados, generando que el interés por participar de la comunidad sea cada día menor. En ese sentido, existe la voluntad de los diferentes actores de sentarse en mesas de trabajo y diálogo, no obstante, no se ha podido abordar una solución a la crisis ambiental, ya que no hay un acuerdo empresarial y político de conciliar el desarrollo con la protección ambiental.

Por una parte, las empresas no esperan dar más de lo que les exige la ley; por otra, el Estado y las industrias no toman participación en el manejo del Pasivo Ambiental y no se promueve el control y regulación acorde a la problemática en la zona, entre otras situaciones. Por lo tanto, las mesas de diálogo buscan soluciones o desafíos menores, que no responden a la solución de un conflicto que requiere de grandes decisiones. En cuanto a ello, se puede identificar que, para dar respuesta a este conflicto, es necesario que comiencen a actuar sobre las bases del problema, con el fin de conciliar el desarrollo local, el cuidado medioambiental y la economía nacional y regional.

También sobre este principio se buscó profundizar sobre la percepción que se tiene sobre las leyes y normas chilenas y su vinculación con el conflicto. En este punto, se puede llegar a la conclusión de que se requiere de una mayor 
exigencia para dar solución a este problema, es decir, se necesitan decisiones políticas más drásticas y concretas, que sean capaces de regularse, fiscalizarse, y también sancionarse. A la vez, se requiere de una mayor voluntad política de escuchar las exigencias locales, situación que se evidencia con años de lucha de diversas comunidades de se manifestaron frente a las débiles normativas ambientales chilenas, que fueron ignoradas y que, sin embargo, tuvieron que ser resueltas por las exigencias de la OCDE, lo que deja inferir la falta de voluntad política para ponerse en el lugar de las comunidades.

\section{Tabla 4}

Principio Conciliador Desarrollo y Cuidado medioambiental

\begin{tabular}{|l|l|l|}
\hline \multicolumn{1}{|c|}{ Empresas } & \multicolumn{1}{|c|}{ Autoridades } & \multicolumn{1}{c|}{ Comunidad } \\
\hline $\begin{array}{l}\text { Se reconoce que han } \\
\text { existido instancias de } \\
\text { dialogo, pero no han dado } \\
\text { buenos resultados. }\end{array}$ & $\begin{array}{l}\text { Han existido instancias de diálogo, } \\
\text { pero faltan recursos para llegar a } \\
\text { buen resultado. }\end{array}$ & $\begin{array}{l}\text { Se indica que existen } \\
\text { instancias de dialogo, pero } \\
\text { que empresas buscan la } \\
\text { fidelización y no la solución } \\
\text { del problema ambiental. }\end{array}$ \\
\hline $\begin{array}{l}\text { Lo anterior, por expectativas } \\
\text { de corto plazo de la } \\
\text { comunidad, frente a } \\
\text { problemas que se resuelven } \\
\text { en el largo plazo, y } \\
\text { expectativas diversas por } \\
\text { parte de los actores. }\end{array}$ & $\begin{array}{l}\text { Comunidad se integra a participar } \\
\text { cada vez más, pero quieren que la } \\
\text { participación sea vinculante. }\end{array}$ & $\begin{array}{l}\text { Se ve doble discurso del } \\
\text { Estado, ya que mientras } \\
\text { promueve mesas, aprueba } \\
\text { nuevos proyectos para } \\
\text { asentar nuevas industrias en } \\
\text { el área. }\end{array}$ \\
\hline $\begin{array}{l}\text { Se reconocen normativas } \\
\text { exigentes, pero que han } \\
\text { llegado tarde a solucionar el } \\
\text { problema local. }\end{array}$ & $\begin{array}{l}\text { En Chile las normas son } \\
\text { adecuadas, pero falta mayor } \\
\text { capacidad de fiscalización y } \\
\text { seguimiento. } \\
\text { Autoridades locales plantean } \\
\text { que normas no son adecuadas. } \\
\text { Normativa ha llegado retrasada. }\end{array}$ & $\begin{array}{l}\text { Lo que más falta es } \\
\text { fiscalización, pero también } \\
\text { normas y leyes más } \\
\text { exigentes. }\end{array}$ \\
\hline - & $\begin{array}{l}\text { Cambios de gobierno influyen en } \\
\text { lisminución de credibilidad. }\end{array}$ & $\begin{array}{l}\text { Se sienten ignorados por el } \\
\text { Estado. }\end{array}$ \\
\hline
\end{tabular}

Fuente: Elaboración propia

\section{Principio de la Solidaridad}

Una de las principales aristas del conflicto socioambiental, se relaciona con el principio de Solidaridad con las generaciones futuras. Desde esta perspectiva, el pasivo ambiental y los sucesivos accidentes industriales han ido deteriorado paulatinamente el territorio, afectando las posibilidades económicas y de diversificación local. Es así como el bien común presenta altos niveles de contaminación que, mientras no se tomen medidas concretas, seguirá este proceso de decadencia. 


\section{Tabla 5}

Principio de la Solidaridad

\begin{tabular}{|l|l|l|}
\hline \multicolumn{1}{|c|}{ Empresas } & \multicolumn{1}{c|}{ Autoridades } & \multicolumn{1}{c|}{ Comunidad } \\
\hline $\begin{array}{l}\text { Existe una reorientación de } \\
\text { la visión y vinculación con } \\
\text { el territorio por parte de las } \\
\text { empresas. }\end{array}$ & $\begin{array}{l}\text { Nueva relación empresas- } \\
\text { comunidad a partir de } \\
\text { conflicto. }\end{array}$ & Acción tardía de las empresas. \\
\hline $\begin{array}{l}\text { Responsabilidad Social } \\
\text { Empresarial (RSE) y política } \\
\text { empresarial ambiental como } \\
\text { estrategia de negocio de } \\
\text { largo plazo. } \\
\text { Involucramiento mediante } \\
\text { profesionales especializados. }\end{array}$ & $\begin{array}{l}\text { RSE ha sido asumida } \\
\text { tardíamente y en función de } \\
\text { contingencias. }\end{array}$ & $\begin{array}{l}\text { Reclaman que RSE está centrada } \\
\text { en proyectos comunitarios y no en } \\
\text { recuperar la calidad ambiental. } \\
\text { RSE se hace como marketing y no } \\
\text { como compensación por el daño } \\
\text { que se ha realizado. }\end{array}$ \\
\hline $\begin{array}{l}\text { Consideran el mejoramiento } \\
\text { de tecnología para }\end{array}$ & $\begin{array}{l}\text { El mejoramiento tecnológico } \\
\text { minimiza los impactos, } \\
\text { optimizar las condiciones de } \\
\text { producción a futuro. }\end{array}$ & $\begin{array}{l}\text { Los estudios indican el daño } \\
\text { ambiental y a la salud de las } \\
\text { personas, aun así, se permite la la } \\
\text { impactos sinérgicos. }\end{array}$ \\
$\begin{array}{ll}\text { instalación de nuevas empresas. } \\
\text { Existe una proyección sin pensar en } \\
\text { la salud de la comunidad. }\end{array}$ \\
\hline
\end{tabular}

Fuente: Elaboración propia

\section{Principio Precautorio y de Ciencias}

Como se ha mencionado, diversos estudios han demostrado el impacto ambiental y social de décadas de funcionamiento del PIV, situación en que todos los actores presentan un consenso. Las empresas y autoridades, por su parte, señalan que el principio Precautorio y de las Ciencias se lleva a cabo mediante las normativas y buenas exigencias ambientales; sin embargo, la comunidad no está de acuerdo con ello, ya que considera la normativa poco exigente y que no resguarda la salud de la comunidad.

Tabla 6

Principio Precautorio y de Ciencias

\begin{tabular}{|l|l|l|}
\hline \multicolumn{1}{|c|}{ Empresas } & \multicolumn{1}{c|}{ Autoridades } & \multicolumn{1}{c|}{ Comunidad } \\
\hline $\begin{array}{l}\text { El mejoramiento de la } \\
\text { calidad ambiental es } \\
\text { evidencia de normativas } \\
\text { buenas y exigentes. }\end{array}$ & $\begin{array}{l}\text { Hay mucho mito y } \\
\text { desinformación en } \\
\text { cuanto a la percepción de } \\
\text { la calidad ambiental. }\end{array}$ & $\begin{array}{l}\text { Se sabe los efectos que causa en la salud } \\
\text { la contaminación ambiental presente; } \\
\text { sin embargo, tenemos normas muy por } \\
\text { debajo de lo que propone la OMS y } \\
\text { carecemos de normas importantes para } \\
\text { resguardar la salud de la comunidad. }\end{array}$ \\
\hline - & $\begin{array}{l}\text { No se incorpora en la } \\
\text { normativa los efectos del } \\
\text { impacto sinérgico. }\end{array}$ & $\begin{array}{l}\text { Están declarados como saturados y aun } \\
\text { así siguen instalando y permiten nuevas } \\
\text { empresas. }\end{array}$ \\
\hline
\end{tabular}

Fuente: Elaboración propia 


\section{Discusión}

Los resultados entregados por este estudio permiten dar luces de algunas claves en la problemática analizada. Para sintetizar lo anterior, en la siguiente tabla se realiza una clasificación de percepciones en función de si estas son positivas o negativas, en caso de que existiese cierta uniformidad en el fondo del tema, así como aquellas en las que se presentan divergencias entre lo visualizado por empresas, autoridades o comunidad.

\section{Tabla 7}

\section{Percepciones}

\begin{tabular}{|c|c|c|}
\hline Percepción & Convergente & Divergente \\
\hline Positiva & $\begin{array}{l}\text { Reconocimiento de un principio de } \\
\text { Responsabilidad de las empresas en el } \\
\text { conflicto. } \\
\text { Se cumple la normativa. } \\
\text { Cada vez existe mayor preocupación } \\
\text { por el medio ambiente. } \\
\text { Relación con la comunidad cada vez es } \\
\text { mejor. } \\
\text { Han existido instancia de diálogo, } \\
\text { aunque no se ha llegado a buenos } \\
\text { resultados. }\end{array}$ & \multirow{2}{*}{$\begin{array}{l}\text { Normativa ambiental es muy exigente } \\
\text { o muy laxa. } \\
\text { Se hacen buenos estudios o son } \\
\text { estudios poco claros y no se prioriza el } \\
\text { medio ambiente. } \\
\text { Tecnología se incorpora o no hay } \\
\text { disposición a hacerlo. } \\
\text { Algunos solo ven vínculos positivos y } \\
\text { desde otro extremo negativos entre } \\
\text { empresas y comunidad. } \\
\text { Salud ambiental de las personas sigue } \\
\text { afectada. }\end{array}$} \\
\hline Negativa & $\begin{array}{l}\text { Nadie se hace cargo del Pasivo } \\
\text { Ambiental. } \\
\text { Incentivo está en lo económico más que } \\
\text { en lo ambiental, generando clientelismo } \\
\text { por parte de las empresas respecto de } \\
\text { las comunidades. } \\
\text { Conflictividad aumenta con llegada de } \\
\text { nuevas empresas, así como los efectos } \\
\text { ambientales sinérgicos. } \\
\text { Discontinuidad en políticas de Estado, } \\
\text { por cambios de gobierno. }\end{array}$ & \\
\hline
\end{tabular}

Fuente: Elaboración propia

En relación a las competencias técnicas, reflejado en la divergencia de visiones existentes respecto de la normativa ambiental, la claridad de los estudios e incorporación tecnológica, Vallejos-Romero et al. (2016), detectan una hallazgo similar en relación con estudios llevados a evaluación ambiental de proyectos energéticos, sobre todo por una baja percepción de las competencias profesionales de los encargados, así como el alcance o limitaciones de las instituciones en relación con experiencia, recursos y capacidades. Este mismo trabajo también muestra el enfoque clientelista de las empresas en relación con las comunidades, centrando la mirada más en explicar los beneficios económicos que los riesgos a los cuales pueda estar expuesta la comunidad. 
También es relevante destacar cómo se evidencia el cumplimiento normativo en cuanto a percepción de todos los actores, pero que en términos reales esto no se verifique en una disminución del conflicto o percepción de mejoras en la calidad ambiental. De forma similar a esto, Cordero (2011) plantea que el ordenamiento jurídico del país en materia ambiental se articula orgánicamente pero no así su efecto: "Hay una separación tajante entre lo que dispone nuestro ordenamiento jurídico y la realidad" (Cordero, 2011: 248). De todas maneras, es importante lo que indica respecto de este punto Contreras (2014), en el sentido de que hay normas que lograron su objetivo, pero están obsoletas, otras cuyos objetivos de protección está lejos del nivel de exigencia, normas que no han sido actualizadas y, finalmente, normas para proteger a las poblaciones vulnerables que no han sido implementadas.

En relación con la contaminación y la permanencia del pasivo ambiental, Bolados y Sánchez (2017) confirman los hallazgos desde una perspectiva feminista, colocando a la mujer como portadoras de las conciencias locales: "Lo anterior es posible a partir de verse afectadas por los pasivos industriales que les impactan a nivel cotidiano, repercutiendo en la salud de su familia, en la contaminación de los cuerpos humanos y el ecosistema" (Bolados y Sánchez, 2017: 40). En el mismo sentido respecto de este Pasivo Ambiental, Hervé et al. (2012) indican que ha sido provocado en sus inicios por empresas del Estado (ENAMI, CODELCO, CHILGENER), no obstante, no se han implementado políticas de reparación ambiental ni de derechos humanos en el área.

También es posible comentar, respecto de la desarticulación de las comunidades, que es un fenómeno reflejado en este estudio y comprobado por Saravia et al. (2016) al analizar el derrame de petróleo en Quintero, y como éste influyó para que existiese un ánimo de movilización en el corto plazo, pero que posteriormente no se transformó en un movimiento capaz de unificar las visiones en el territorio.

\section{Conclusiones}

En función del estudio realizado, es posible identificar que la DSI, vista desde una perspectiva práctica, como una herramienta para analizar, en este caso, un conflicto ambiental, es de utilidad, puesto que permite descomponer la problemática en distintos enfoques de análisis que, siendo diferentes, son complementarios.

En el contexto anterior el estudio permite indicar que el principio de la Responsabilidad y el deber de Tutela, tienen un grado muy bajo de implementación, puesto que, más allá de que se evidencien efectos ambientales y sobre todo el Pasivo Ambiental existente, los que afectan a la comunidad, no han existido soluciones concretas para resolver el problema en su conjunto, 
y más bien los mismos actores perciben el actuar del Estado y las empresas como soluciones parciales y reactivas a los eventos de contingencia.

Respecto del principio de la Ecología Integral, su aplicación en relación a la comunidad ha sido equívoco, ya que se ha basado en la idea de generar fidelidad y licencia social, más que abrir un diálogo y apuntar a soluciones concretas al conflicto. Esto se ha traducido en que los programas de responsabilidad social implementados por cada empresa de forma individual han provocado el socavamiento de las relaciones comunitarias, debido a la generación de grupos de interés que funcionan de forma satélite a cada una de ellas, los cuales, en momentos de ocurrir alguna contingencia ambiental, defienden a la empresa responsable del evento debido a los beneficios que les otorga, mientras el resto de la comunidad exige soluciones, y en ese mismo sentido la comunidad finalmente no percibe una mejora en sus condiciones de calidad de vida.

De todas maneras, para cerrar este artículo, es relevante indicar que, con el tiempo, se han dado percepciones positivas convergentes que se vinculan con el reconocimiento de las empresas en relación con el conflicto, la mayor preocupación por el medio ambiente y que la relación con la comunidad es cada vez mejor, lo que da luces de esperanza para mejoras en las situaciones actuales en las que viven.

\section{Referencias bibliográficas}

\section{Fuentes Primarias}

Pablo VI (1967). Carta encíclica "Populorum Progressio. Revisado el 2-I-2018, en http://w2.vatican.va/content/paul-vi/es/encyclicals/documents/hf_p-vi_ enc_26031967_populorum.pdf

Conferencia Episcopal Paraguaya (1983). Carta Pastoral El campesino paraguayo y la tierra. Revisado el 2-I-2018, en http://episcopal.org.py/news-item/el-campesinoparaguayo-y-la-tierra-12-de-junio-de-1983/

Juan Pablo II (1988). Exhortación Apostólica Postsinodal Christifideles Laici. Revisado el 2-I-2018, en http://W2.vatican.va/content/john-paul-ii/es/apost_exhortations/ documents/hf_jp-ii_exh_30121988_christifideles-laici.html

Juan Pablo II (1990). Mensaje de Su Santidad para la celebración de la XXIII Jornada Mundial de la Paz. Revisado el 2-I-2018, en https://w2.vatican.va/content/ john-paul-ii/es/messages/peace/documents/hf_jp-ii_mes_19891208_xxiii-worldday-for-peace.html

Juan Pablo II (1991). Carta Encíclica Centesimus Annus. Revisado el 2-I-2018, en http://w2.vatican.va/content/john-paul-ii/es/encyclicals/documents/hf_jpii_enc_01051991_centesimus-annus.html 
Juan Pablo II (1995). Carta encíclica Evangelium Vitae. Revisado el 2-I-2018, en http://w2.vatican.va/content/john-paul-ii/es/encyclicals/documents/hf_.jpii_enc_25031995_evangelium-vitae.html

Juan Pablo II (1997). Discurso del Santo Padre Juan Pablo II a los promotores y participantes en un Congreso Internacional sobre "Ambiente y Salud". Revisado el 2-I-2018, en https://w2.vatican.va/content/john-paul-ii/es/speeches/1997/march/ documents/hf_jp-ii_spe_19970324_ambiente-salute.html

Juan Pablo II (1999a). Exhortación Apostólica Postsinodal Ecclesia in Asia. Revisado el 2-I-2018, en http://w2.vatican.va/content/john-paul-ii/es/apost_exhortations/ documents/hf_jp-ii_exh_06111999_ecclesia-in-asia.html

Juan Pablo II (1999b). Exhortación Apostólica Postsinodal Ecclesia in América. Revisado el 2-I-2018, en http://w2.vatican.va/content/john-paul-ii/es/apost_exhortations/documents/hf_jp-ii_exh_22011999_ecclesia-in-america.html

Juan Pablo II (2002). Declaración Conjunta del Santo Padre Juan Pablo II y su santidad Bartolomé I. Firma de la Declaración de Venecia. Revisado el 2-I-2018, en https://w2.vatican.va/content/john-paul-ii/es/speeches/2002/june/documents/ hf_jp-ii_spe_20020610_venice-declaration.html

Juan Pablo II (2003). Exhortación Apostólica Postsinodal Pastores Gregis. Revisado el 2-I-2018, en http://w2.vatican.va/content/john-paul-ii/es/apost_exhortations/ documents/hf_jp-ii_exh_20031016_pastores-gregis.html

Juan Pablo II (2004). Compendio de la Doctrina Social de la Iglesia. Revisado el 2-I-2018, en http://www.vatican.va/roman_curia/pontifical_councils/justpeace/ documents/rc_pc_justpeace_doc_20060526_compendio-dott-soc_sp.html

Juan Pablo II (2004). Mensaje del Santo Padre Juan Pablo Il para la Jornada Mundial de la Alimentación. Revisado el 2-I-2018, en https://w2.vatican.va/content/ john-paul-ii/es/messages/food/documents/hf_jp-ii_mes_20041015_xxiv-worldfood-day.html

Benedicto XVI (2009). Caritas in Veritate. Vaticano: Editrice Vaticana.

Benedicto XVI (2009). Mensaje de Su Santidad Benedicto XVI para la celebración de la XLII Jornada Mundial de la Paz. Revisado el 2-I-2018, en http://w2.vatican.va/ content/benedict-xvi/es/messages/peace/documents/hf_ben-xvi_mes_20081208_ xlii-world-day-peace.html

Benedicto XVI (2010). Mensaje de Su Santidad Benedicto XVI para la celebración de la XLIII Jornada Mundial de la Paz. Revisado el 2-I-2018, en https:// w2.vatican.va/content/benedict-xvi/es/messages/peace/documents/hf_benxvi_mes_20091208_xliii-world-day-peace.html

Francisco (2013). Audiencia General 5-VI-2013. Revisado el 2-I-2018, en https:// W2.vatican.va/content/francesco/es/audiences/2013/documents/papa-francesco_20130605_udienza-generale.pdf 
Francisco (2015). Laudato Si' Revisado el 2-I-2018, en http://w2.vatican.va/ content/francesco/es/encyclicals/documents/papa-francesco_20150524_enciclicalaudato-si.pdf

\section{Fuentes Secundarias}

\section{a) Artículos y capítulos de libros}

Bolados, P. A. y Sánchez, A. (2017). "Una ecología política feminista en construcción: el caso de las 'Mujeres de zonas de sacrificio en resistencia', Región de Valparaíso, Chile", en Psicoperspectivas, Vol. 16, № 2, pp. 33-42.

Cordero, E. (2011). "Ordenamiento territorial, justicia ambiental y zonas costeras", en Revista de derecho, №36, pp. 209-249.

Correa, J. (2012). "Complejo Industrial Ventanas: Exclusión de la Ciudadanía y Problemas de Gobernabilidad. El Caso 'Campiche' entre lo Legal y lo Legítimo", en Justicia Ambiental, Vol. IV, Nº 4, pp. 121-136.

De Gregori, I., E. Fuentes, M. Rojas, H. Pinochet y M. Potin-Gautier (2003). "Monitoring of copper, arsenic and antimony levels in agricultural soils impacted and non-impacted by mining activities, from three regions in Chile", en Journal of environmental monitoring, Vol. 5, № 2, pp. 287-295.

Fuenzalida, M., y R. Quiroz (2012). "La dimensión espacial de los conflictos ambientales en Chile", en Polis, Vol. 11, № 31, pp. 157-168.

Ginocchio, R. (2000). "Effects of a copper smelter on a grassland community in the Puchuncavi Valley, Chile", en Chemosphere, Vol. 41, № 1-2, pp. 15-23.

Gonzalez, I., A. Neaman, P. Rubio y A. Cortes (2015). "Spatial distribution of copper and pH in soils affected by intensive industrial activities in Puchuncavi and Quintero, central Chile", en Journal of soil science and plant nutrition, Vol. $14, N^{\circ} 4$, pp. 943-953.

Hervé, D., J. Schönsteiner, S. Mariangel, y I. Mewes (2012). "Empresas, Medio Ambiente y Derechos Humanos: La Zona Industrial de Quintero-Puchuncavi", en A. Coddou, y S. Missana, Informe anual sobre derechos humanos en Chile 2012. Santiago de Chile: Universidad Diego Portales, pp. 131-161.

Neaman, A., L. Reyes, F. Trolard, G. Bourrié, y S. Sauvé (2009). "Copper mobility in contaminated soils of the Puchuncaví valley, central Chile", en Geoderma, № 150, pp. 359-366.

Oyarzún, M. (2010). "Contaminación aérea y sus efectos en la salud", en Revista chilena de enfermedades respiratorias, Vol. 26, № 1, pp. 16-25.

Parra, S., M. Bravo, W. Quiroz, T. Moreno, A. Karanasiou, O. Font y F. Cereceda (2014). "Distribution of trace elements in particle size fractions for contaminated 
soils by a copper smelting from different zones of the Puchuncavi Valley (Chile)", en Chemosphere, № 111, pp. 513-521.

Sabatini, F., F. Mena y P. Vergara (1997). "Otra vuelta a la espiral: El conflicto ambiental de Puchuncaví bajo la democracia" en F. Sabatini, y C. Sepúlveda, Conflictos Ambientales. Entre la Globalización y la Sociedad Civil. Santiago: CIPMA, pp. 107-140.

Salmanighabeshi, S., M. Palomo-Marin, E. Bernalte, F. Rueda-Holgado, C. MiroRodriguez, X. Fadic-Ruiz y E. Pinilla-Gil (2015). "Long-term assessment of ecological risk from deposition of elemental pollutants in the vicinity of the industrial area of Puchuncavi-Ventanas, central Chile", en Science of the total environment, $N^{\circ} 527$, pp. 335-343.

Saravia, P., K. Armigol y B. Garland (2016). "El derrame de petróleo en Quintero, $\checkmark$ región de Chile. Una mirada desde las organizaciones sociales", en Población y sociedad. Vol. 23, N² 2, pp. 179-206.

Siegel, E. y P. Siegel (1995). "ENAMI's Ventanas smelter heavy metals pollution", en International Journal of Surface Mining, Reclamation and Environment, Vol. 9, $\mathrm{N}^{\circ}, 2$, pp. 79-82.

Vallejo, J. (2012). "Bahía de Quintero: Zona de Sacrificio Un aporte desde la Justicia Ambiental", en Publicaciones Fundación Terram, № 53, pp. 1-35.

Vallejos-Romero, A., A. Boso y H. Zunino (2016). "La relevancia de la confianza en conflictos socioambientales por energía en Chile: los casos de 'Castilla' e 'Hidroaysén", en Revista de Geografía Norte Grande, № 63, pp. 145-162.

\section{b) Libros}

Consejo Pontificio Justicia y Paz (2004). Compendio de la doctrina social de la iglesia. Ciudad del Vaticano: Editrice Vatican.

Observadores de Derechos Humanos, Casa Memoria José Domingo Cañas. (2013). Informe de observación: Puerto Ventanas- Puchuncaví Región de Valparaíso. Santiago, Chile: Fundación Casa Memoria José Domingo Cañas.

Observatorio Latinoamericano de Conflictos Ambientales, OLCA (2012). Agua y extractivismo: Una mirada desde las comunidades. Santiago, Chile: OLCA.

\section{c) Tesis}

Calisto, M. (2014). Observatorio del Medioambiente Las Ventanas. Proyecto de Título para optar al grado de Arquitecto, Universidad de Chile.

Sánchez, E. (2013). Plan Maestro de Regeneración del Borde Costero de Ventanas. Proyecto de Título para optar al grado de Arquitecto, Universidad de Chile. 


\section{d) Otros}

Comisión de Recursos Naturales, Bienes Nacionales y Medio Ambiente. (2011). Informe de la comisión de recursos naturales, bienes nacionales y medio ambiente recaído en el mandato otorgado por la sala a fin de analizar, indagar, investigar y determinar la participación de la empresa estatal CODELCO y empresas asociadas, en la contaminación ambiental en la zona de Puchuncaví y Quintero.

Contreras, C. (2014). Problemas de salud en Ventanas: ¿por qué existen si la zona cumple con la normativa ambiental? Oportunidad para mejores regulaciones ambientales a partir de directrices OCDE. Santiago: s/e.

ONG Oceana. (2012), http://oceana.org/es/sa/nuestro-trabajo/energia-limpia/ zonas-de-sacrificio/mas-informacion/zonas-de-sacrificio-medicion-de-metalesen-mariscos-y-crus, Revisado el 28-XII-2015. 